\title{
Age-Specific Clinical Features of Erectile Dysfunction
}

\author{
Liuhong Cai, Manbo Jiang, Minhui Zeng, Weijie Xing, Yanfei Wen, Bin Zhang* \\ Department of Infertility and Sexual Medicine, The Third Affiliated Hospital, Sun Yat-Sen University, Guangzhou, \\ China \\ Email: ${ }^{\text {doc305@163.com }}$
}

Received 25 February 2014; revised 29 March 2014; accepted 8 April 2014

Copyright @ 2014 by authors and Scientific Research Publishing Inc.

This work is licensed under the Creative Commons Attribution International License (CC BY).

http://creativecommons.org/licenses/by/4.0/

(c) (i) Open Access

\section{Abstract}

Sexual health can be considered as a mirror of general health and, in turn, general health is a prerequisite for sexual heath. The aim of this study was to compare the different clinical features of erectile dysfunction (ED) in young and elderly males. From June 2011 to December 2011, 224 patients were included and divided into two groups. Elderly ED group consisted of 120 patients with ED and all were 61 - 90 years old, and young ED group consisted of 104 patients with ED and all were 21 - 45 years old. Questionnaires, physical examination, blood sample test, ultrasound monitor, time of achieving erection (TAE), intra-vaginal ejaculation latency time (IELT), ECG and penis-brachial blood pressure index (PBI) were investigated. There were significant differences between the two groups, regarding the top three complications, the body mass index (BMI), International Index of Erectile Function-5 (IIEF-5) scores, Self-rating Depression Scale (SDS) total scores, TAE, IELT and PBI. We concluded that clinical features of ED were age-specific, which will be helpful for treatment strategies and evaluation of the treatment efficacy.

\section{Keywords}

Elderly Male, Erectile Dysfunction, The Time of Achieving Erection, Intra-Vaginal Ejaculatory Latency Time, Depression

\section{Introduction}

Sexual health can be considered as a mirror of general health and, in turn, general health is a prerequisite for sexual heath. Though Masters and Johnson found no upper age limit for sexual function and many elderly men continue to be sexually active throughout their lives [1], a 9-year period study showed that within-person change

Corresponding author. 
in all aspects of sexual function was strongly related to age [2]. Erectile dysfunction (ED) is now well-studied and thought to be a symptom of underlying disease rather than a disease itself [3]. The role of the endothelium in regulating erectile physiology is well established, and the incidence of ED increases with age, usually associating with changes in arterial structure and function [4]-[7]. People are living longer and healthier lives nowadays, and elderly people proportion in this society is increasing. Elderly males will be different from young males in hormone level, general body and psychologic condition, sexual arousal, underlying diseases, and so on. It is important to distinguish the different clinical features of ED for young and elderly males for the treatment purpose. However, no sufficient studies regarding this aspect will be helpful for treatment strategies and evaluation of the treatment efficacy.

\section{Materials and Methods}

\subsection{Ethical Approval}

All the subjects were given a thorough explanation of the study and a written informed consent was obtained before participating in the study. Approval from the Reproductive Ethics Committee of The Third Affiliated Hospital, Sun Yat-Sen University, was obtained.

\subsection{Participants}

From June 2011 to December 2011, male patients visited Department of Infertility and Sexual Medicine, The Third Affiliated Hospital, Sun Yat-Sen University. Inclusion criteria: 1) main complaints of ED at least for 6 months; 2) age of 61 to 90 years old or 21 to 45 years old; 3) Evaluated by International Index of Erectile Function-5 (IIEF-5) to confirm an ED diagnosis. Exclusion criteria: Patients diagnosed as traumatic ED. Those who did not complete all of the examinations and questionnaires were also excluded.

\subsection{Methods}

1) After a detailed medical history was obtained, all of the patients filled in the IIEF-5 questionnaires and Self-rating Depression Scale (SDS) and a self-designed questionnaire for sociodemographic data independently.

2) All patients implemented physical examination (including calculation of body mass index, BMI), rectal examination, blood sample test for fasting blood glucose, ultrasound monitor for prostate and heart, and ECG. If there were abnormal results, the patient was arranged to see a specialist for diagnosis and treatment.

3) Penis-brachial blood pressure index (PBI) was calculated by the formula: penile arterial blood pressure/ brachial blood pressure. Doppler ultrasound monitor instrument was used to measure the penile cavernous artery systolic pressure on both sides, and then measure the brachial artery systolic pressure.

4) All of the patients were provided with a stopwatch and instructed how to record the time of achieving erection (TAE) and intra-vaginal ejaculation latency time (IELT). Premature ejaculation (PE) was diagnosed basing on ISSM criteria [8].

\subsection{Statistical Analysis}

Statistical analysis was performed by using the computer statistical package SPSS/10.0 (Chicago, IL, USA). A p-value less than 0.05 was considered statistically significant. Continuous variables were expressed as $\bar{x} \pm s$, for normally distributed variables, t-test was used. As the IELT in individual patient usually follows a skewed distribution, we used the Wilcoxon rank to calculate geometric mean IELTs instead of mean IELTs. $\chi^{2}$ test was used for classification variables

\section{Results}

An experienced technician was responsible for data collection. Totally 250 patients were recruited, and 26 were excluded due to incomplete data, 224 (89.6\%) cases were included in the study. Based on their age, the studied population were divided as elderly male ED group (elderly ED group, 61 to 90 years old) and young male ED group (young ED group, 21 to 45 years old). Table 1 showed the socio-demographic data and comparison of the clinical features between the two groups. 
Table 1. Comparison of the two groups.

\begin{tabular}{|c|c|c|c|c|}
\hline Characteristics & & Elderly ED group & Young ED group & $P$ value \\
\hline Age (years) & & $65.9 \pm 10.2$ & $36 \pm 6.6$ & $\mathrm{P}<0.05$ \\
\hline Education & Illiterate & 5 & 1 & $\mathrm{P}<0.05$ \\
\hline \multirow[t]{3}{*}{ (cases) } & Primary education & 33 & 7 & \\
\hline & High school & 65 & 34 & \\
\hline & University education & 17 & 70 & \\
\hline BMI & & $30.9 \pm 6.1$ & $25.4 \pm 2.3$ & $\mathrm{P}<0.05$ \\
\hline IIEF-5 scores & & $14.82 \pm 4.17$ & $11.83 \pm 3.67$ & $\mathrm{P}<0.05$ \\
\hline Complications & Cardiovascular Disease & 65 & 3 & $\mathrm{P}<0.05$ \\
\hline \multirow[t]{5}{*}{ (cases) } & Prostatic hyperplasia & 48 & 12 & \\
\hline & Diabetes & 40 & 5 & \\
\hline & Chronic prostatitis & 33 & 66 & \\
\hline & Premature Ejaculation & 35 & 41 & \\
\hline & Depression & 2 & 18 & \\
\hline SDS total scores & & $29.13 \pm 5.63$ & $39.59 \pm 13.31$ & $\mathrm{P}<0.05$ \\
\hline TAE (minutes) & & $13.85 \pm 5.75$ & $3.61 \pm 4.29$ & $\mathrm{P}<0.05$ \\
\hline IELT (minutes) & & $7.03 \pm 5.35$ & $3.81 \pm 5.53$ & $\mathrm{P}<0.05$ \\
\hline PBI & & $0.78 \pm 0.12$ & $0.91 \pm 0.06$ & \\
\hline
\end{tabular}

\section{Discussion}

ED patients had multiple complications, in an age-specific manner. ED is now well-studied and thought to be a symptom of underlying disease rather than a disease itself [3]. In this study, top three complications of elderly ED group were cardiovascular disease (CVD), benign prostatic hyperplasia (BPH) and diabetes, but for young ED group, the top three complications were chronic prostatitis, premature ejaculation (PE) and depression.

Studies showed that men over 65 years of age, testosterone deficiency seems to play an important role, the degree of ED was significantly higher in men with lowest testosterone levels [9]. Due to aging, elderly males are also affected by multiple organic diseases which could play a crucial role in the pathogenesis of dysfunction. As we know, the underlying processes in organic ED are arterial insufficiency, vascular occlusion disease, or combinations of both, and ED and CVD should be regarded as two different manifestations of the same systemic disorder [10]. Diabetes leads to penile cavernous neurovascular degeneration, resulting in reduced number of corpus cavernous smooth muscle, and the formation of ED affect cavernous expansion. On the other hand reduced compliance of cavernous smooth muscle relaxation results in the outflow of blood vessels of the penis to form secondary latch dysfunction venous ED. Both CVD and diabetes are age-related diseases. These evidences suggested that many elderly ED may be a local manifestation of systemic disease.

Studies showed strong evidence that lower urinary tract symptoms (LUTS) and ED are correlated, although a direct causal relationship has not been established yet [11]. There was an association between ED and having been previously diagnosed with chronic prostatitis/chronic pelvic pain syndrome (CP/CPPS). Urologists should be alert to the association between CP/CPPS and ED, and assess the ED patients suffering from CP/CPPS [12]. But difference still existed between the two groups, for elderly ED group, it was benign prostatic hyperplasia and chronic prostatitis for young ED group as one of the top three complications.

Study has shown that aging was correlated with less anxiety and more sexual symptoms [13], we also found this interesting phenomena in this study that less depression in elderly ED group, only 2 in 120, but it was 18 in 104 for young ED group. Not surprisingly, SDS scores were $29.13 \pm 5.63$ in elderly ED group and $39.59 \pm 13.31$ in young ED group $(\mathrm{P}<0.05)$. It may be due to the fact that most elderly males treated ED as a natural manifestation. In other words, it may also demonstrate the truth that the proportion of psychogenic ED is lower in elderly ED group than in young ED group.

ED patients had different clinical manifestations, also in an age-specific manner. In this study, IIEF scores showed moderate ED in both groups, with scores in elderly ED group a little higher than in young ED group ( $P$ 
$<0.05)$.

PBI measurement is a diagnostic modality useful in elucidating the cause of ED and the magnitude of its severity. $\mathrm{PBI}$ is the ratio of arterial pressure and the pressure of the dorsal penile brachial artery, and is used to evaluate penile blood supply. Normal PBI value should be above 0.8. In this study, PBI value of the elderly ED group was significantly lower than that of the young group $(\mathrm{P}<0.05)$, supporting the fact that cardiovascular disease was a major cause for elderly ED.

The time of achieving erection (TAE) is the time required for penis to reach full erection for penetration, which is helpful for objective assessment of penile erectile function. In this study, the elderly ED group has significantly longer TAE than the young ED group (13.85 \pm 5.75 minutes vs $3.61 \pm 4.29$ minutes). In addition to the organic disease of penis, poor general condition, decreased sexual arousal and decreased erectile central function result in delayed TAE in elderly ED group to attain a similar response as young ED group.

\section{Conclusion}

In all, ED patients had multiple complications and different clinical manifestations in an age-specific manner. Sexual attempts were an age- and testosterone-dependent phenomenon, and sexual function declining with body functions is a natural physiological and pathological process for elderly males [14]. Distinguishing the clinical features of ED related to age will be helpful for treatment strategies and evaluation of the treatment efficacy. However, the sample size of this study is not large enough, which may cause statistical bias.

\section{References}

[1] Masters, M.H. and Johnson, V. (1966) Human Sexual Response. Little Brown \& Co., Boston.

[2] Araujo, A.B., Mohr, B.A. and McKinlay, J.B. (2004) Changes in Sexual Function in Middle-Aged and Older Men: Longitudinal Data from the Massachusetts Male Aging Study. Journal of the American Geriatrics Society, 52, 15021509. http://dx.doi.org/10.1111/j.0002-8614.2004.52413.x

[3] Yao, F., Liu, L., Zhang, Y., Huang, Y., Liu, D., Lin, H., Liu, Y., Fan, R., Li, C. and Deng, C. (2013) Erectile Dysfunction May Be the First Clinical Sign of Insulin Resistance and Endothelial Dysfunction in Young Men. Clinical Research in Cardiology, 102, 645-651. http://dx.doi.org/10.1007/s00392-013-0577-y

[4] Traish, A.M. and Galoosian, A. (2013) Androgens Modulate Endothelial Function and Endothelial Progenitor Cells in Erectile Physiology. Korean Journal of Urology, 54, 721-731.

[5] Aversa, A., Bruzziches, R., Francomano, D., Natali, M., Gareri, P. and Spera, G. (2010) Endothelial Dysfunction and Erectile Dysfunction in the Aging Man. International Journal of Urology, 17, 38-47. http://dx.doi.org/10.1111/j.1442-2042.2009.02426.x

[6] Hamed, S., Brenner, B. and Roguin, A.(2011) Nitric Oxide: A Key Factor behind the Dysfunctionality of Endothelial Progenitor Cells in Diabetes Mellitus Type-2. Cardiovasc Research, 91, 9-15. http://dx.doi.org/10.1093/cvr/cvq412

[7] Haas, C.A., Seftel, A.D., Razmjouei, K., Ganz, M.B., Hampel, N. and Ferguson, K. (1998) Erectile Dysfunction in Aging: Upregulation of Endothelial Nitric Oxide Synthase. Urology, 51, 516-522. http://dx.doi.org/10.1016/S0090-4295(97)00715-2

[8] McMahon, C.G., Althof, S.E., Waldinger, M.D., et al. (2008) An Evidence-Based Definition of Lifelong Premature Ejaculation: Report of the International Society for Sexual Medicine (ISSM) ad hoc Committee for the Definition of Premature Ejaculation. Journal of Sex Medicine, 5, 1590-1606. http://dx.doi.org/10.1111/j.1743-6109.2008.00901.x

[9] Rabijewski, M., Papierska, L., Kozakowski, J. and Zgliczyński, W. (2012) The High Prevalence of Testosterone Deficiency in Population of Polish Men over 65 Years with Erectile Dysfunctions. Aging Male, 15, 258-262. http://dx.doi.org/10.3109/13685538.2012.729233

[10] Gandaglia, G., Briganti, A., Jackson, G., Kloner, R.A., Montorsi, F., Montorsi, P. and Vlachopoulos, C. (2013) A Systematic Review of the Association Between Erectile Dysfunction and Cardiovascular Disease. European Urology, [Epub ahead of print].

[11] Orabi, H., Albersen, M. and Lue, T.F. (2011) Association of Lower Urinary Tract Symptoms and Erectile Dysfunction: Pathophysiological Aspects and Implications for Clinical Management. International Journal of Impotence Research, 23, 99-108. http://dx.doi.org/10.1038/ijir.2011.14

[12] Chung, S.D., Keller, J.J. and Lin, H.C. (2012) A Case-Control Study on the Association between Chronic Prostatitis/ Chronic Pelvic Pain Syndrome and Erectile Dysfunction. BJU International, 110, 726-730. http://dx.doi.org/10.1111/j.1464-410X.2011.10807.x

[13] Chen, C.Y., Lee, C.P., Chen, Y., Jiang, J.R., Chu, C.L. and Chen, C.L.(2013) The Correlation between Emotional Dis- 
tress and Aging Males’ Symptoms at a Psychiatric Outpatient Clinic: Sexual Dysfunction as a Distinguishing Characteristic between Andropause and Anxiety/Depression in Aging Men. Clinical Interventions in Aging, 8, 635-640. http://dx.doi.org/10.2147/CIA.S45190

[14] Corona, G., Rastrelli, G., Monami, M., Maseroli, E., Jannini, E.A., Balercia, G., Sforza, A., Forti, G., Mannucci, E. and Maggi, M. (2013) Frequency of Sexual Activity and Cardiovascular Risk in Subjects with Erectile Dysfunction: CrossSectional and Longitudinal Analyses. Andrology, 1, 864-871. http://dx.doi.org/10.1111/j.2047-2927.2013.00139.x

\section{List of the Abbreviations}

ED Erectile Dysfunction

IIEF-5 International Index of Erectile Function-5

TAE Time of Achieving Erection

PBI Penis-Brachial Blood Pressure Index

BMI Body Mass Index

SDS Self-Rating Depression Scale

PE Premature Ejaculation

CVD Cardiovascular Disease

BPH Benign Prostatic Hyperplasia 